\title{
Electromagnetic Field Released in Collision-Impact Events Generate in the Matrix Interface Fractal Scalable Invariant Geometric Triangular Chiral Hexagonal Structures
}

\author{
Jairo A. Diaz \\ Department of Pathology, Medicine School, Laboratory of Pathology, Hospital Department of Villavicencio, \\ Hospital Department of Granada, University Cooperative of Colombia, Villavicencio, Colombia \\ Email: jaditod@hotmail.com
}

Received April 12, 2013; revised May 20, 2013; accepted June 20, 2013

Copyright (C) 2013 Jairo A. Diaz. This is an open access article distributed under the Creative Commons Attribution License, which permits unrestricted use, distribution, and reproduction in any medium, provided the original work is properly cited.

\begin{abstract}
In biology, cancer is the most beautiful natural model of a chaotic system, which under uncontrolled proliferations, generates extreme disorder that finally causes intercellular collisions. The authors have described and documented fractal self-assembly of geometric triangular chiral hexagonal crystal-like complex organizations (GTCHC) and interface comet tail effect patterns in cancer processes. According to this novel observation cancer incorporates a real visualization world with a great surprising finding in biology, physics, and geology. This visualization platform literally allows us to see what would otherwise remain completely invisible. From theory to practice this irreducible geometric matrix allowed us to identify in geology, real measurable green infrared-electromagnetic stripe line in interface with hexagonal geomorphic pattern, triangular chiral pyramidal rock structures, geology well defined mirror images, template platform to bio signature characterization of ancestral primitive polar head-tail organization, embryoid and human-like shape pattern embedded as giant fossils in rocks that have never been seen before. Electromagnetic field released in collisionimpact events generate in the matrix interphase fractal scalable invariant order of geometric triangular chiral hexagonal structures. The laws of biology and geology can finally be redirected to the laws of physics; specifically magnetic fields create a new kind of classification based on these fractal structural similarities of the relationship. Further interdisciplinary collaboration must be carried out to study these geometric self-assembly geological structures, ancient sediments and rocks that could provide insights into antecedents of life.
\end{abstract}

Keywords: Electromagnetic Fields; Geomorphic Patterns; Irreducible Geometric Matrix

\section{Introduction}

All systems whose particles are in continuous dynamic movement are submitted to laws of physics. This means that during the space-time interval of the life of any system, there exists the absolute potential to enter into a collision state at a given moment.

A collision is an isolated event where two or more moving bodies (colliding bodies) exert forces on each other for a relatively short time. This represents the peak threshold state of a disordered system. Successful collisions have sufficient energy (activation energy) at the moment of impact to break the existing bonds and form new bonds, resulting in the products of the reaction.

In biology, cancer is the most beautiful natural model of a chaotic system, which, under uncontrolled proliferations, generates extreme disorder that finally causes intercellular collisions.

Based on documentary evidence intercellular cancer collision events generate geometry. We described and documented the self-assembly of geometric triangular chiral hexagon crystal-like complex organizations (GTCHC) in human pathological tissues at macroscopic and microscopic levels, mainly in cancer processes [1]. In our experimental model, we were able to reproduce and predict polarity, chirality, helicoid geometry, and triangular and hexagonal clusters through electromagnetic sequential collisions.

Additionally, authors also documented and gathered insights into the magnetic field (MF) in cancer tissues 
and how it generates a functional geometric attractor complex in their entangled state. This geometry occurs on documented collider partners, which are pairs of spiral subpatterns twisted in opposite directions, generating powerful electromagnetic forces during this rotational movement [2]. In our electro-optic collision model, we were able to predict and replicate all the characteristics of this complex geometry that connects a physical phenomenon with the signal patterns that generate biological chaos. Intrinsically, fractal geometry-related collision events make spatial correction errors embrace the chaotic system in a way that permits new structures to emerge, and as a result, an ordered self-assembly of hyper-order domains embryoid bodies with neural differentiation at the final stage of cancer development is a predictable process $[3,4]$.

Recent scientific literature in this research area endorses and is consistent with our findings. The UCLA research group led by Thomas G. Mason, a professor of chemistry and physics as well as a member of the California NanoSystems Institute at UCLA, explored how and why chirality arises. They used a manufacturing technique called lithography, which is the basis for making computer chips, to make millions of microscale particles in the shape of chiral triangles. Using optical microscopy, the researchers then studied very dense systems of these lithographic triangular particles, and to their surprise, they discovered that the chiral triangles spontaneously arranged themselves to form two-triangle "super-structures" with each superstructure exhibiting a particular chirality. In dense systems, entropic electromagnetic forces and particle triangular shape are adequate to cause spontaneous appearance of chirality [5]. Astrophysics from Princeton University, Osaka University and Oxford University, with laboratory experiments discovery-self-organized electromagnetic field structures in laser-produced counter-streaming plasmas [6]. Comparative image analysis of these structures with GTCHC complexes documented in intercellular cancer collisions, reveal absolutely great similarity (Figures 1 and 2). In science when a result obtained in one laboratory is replicated and reproduced in another one with the same results this new knowledge should be incorporated into the academy.

If we could recognize a pattern with clear geometric structuring in a chaotic system and know that the geometry is fractal, governed by the universal laws of physics, then our interest here is to identify similar assemblage patterns at a megascopic level related to geology collision impact events; geometry is an important criterion in determining whether a reaction will take effect in a collision system.

Collision geometry its present at universal scaling and is independent of the nature of colliding systems, further
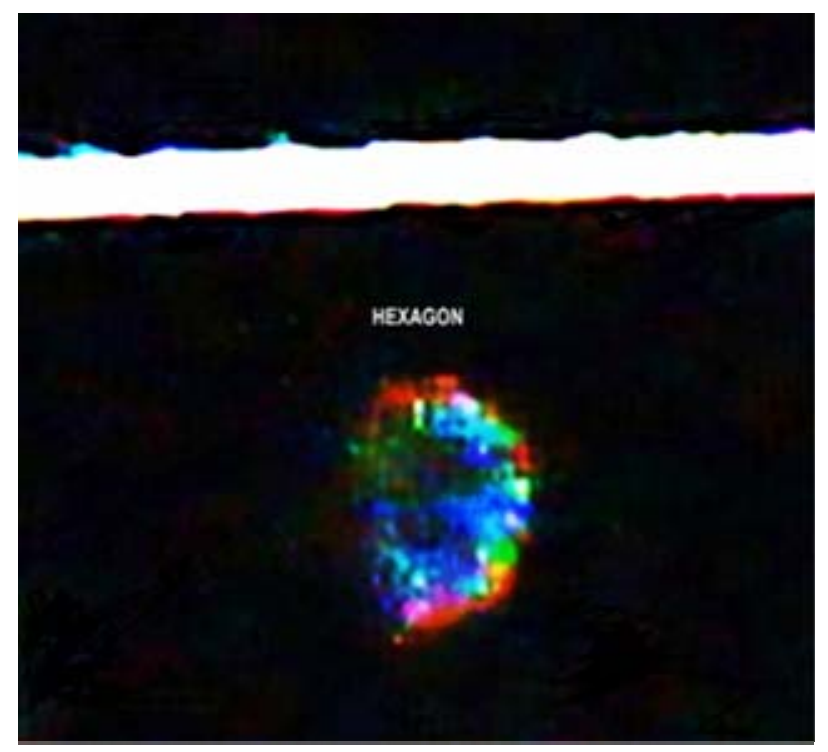

Figure 1. Observation of hexagonal light cluster through electromagnetic sequential collisions.

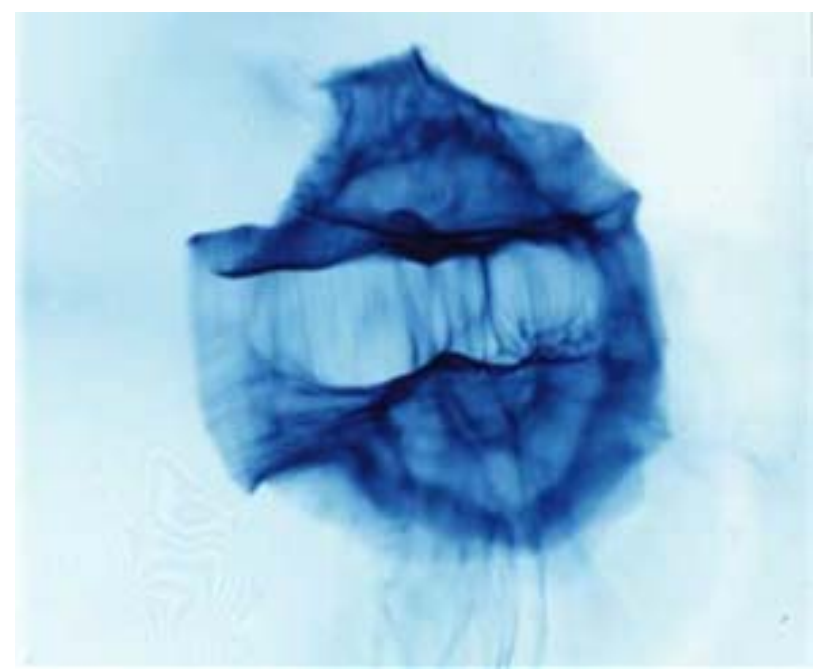

Figure 2. Self-organized electromagnetic field structure in laser-produced counter-streaming plasmas. Particles clusters express hexagonal pattern identical to those documented in our study.

in relation to earth impact events there are not evidence in the current earth sciences literature, about self-assembly of Geometric Triangular Chiral Hexagon Crystal-like complex organizations (GTCHC) in relation to geology impact events, in this way interdisciplinary collaboration in research leads to new solutions to problems through the integration of knowledge-a particular finding in a field of research can complement solutions under new perspectives in another field of knowledge.

\section{Materials and Methods}

Remote sensing is the acquisition of information about 
an object or phenomenon, without making physical contact with the object. In modern usage, the term generally refers to the use of aerial sensor technologies to detect and classify objects on earth, both on the surface and in the atmosphere and oceans. Satellite platforms, such as Google Earth, are valid scientific tools at the disposal of researchers in multiple disciplines. From ruins to the earth impact events, Geologist Arthur Hickman was utilizing Google Earth to find iron ore in Western Australia, when he suddenly found a strange surface. He sent the picture to his colleagues at the Australian National University who visited that area, and consequently, Hickman crater was named [7].

A rayed impact crater of 45-m diameter on a Cretaceous sandstone target was detected in southern Egypt. The ejecta rays highlighted the exceptional freshness of the structure. The crater was identified by V. De Michele during a Google Earth survey and was named as Kamil. A geophysical expedition undertaken in February 2010 revealed that the crater is bowl shaped and has an upraised rim of $3 \mathrm{~m}$ above the pre-impact surface, typical of simple craters [8]. A research team has developed methods that are being used to classify outbreaks, allowing scientists to identify individual organisms that are spreading in the population; using Google Earth, the outbreaks can be easily visualized. The team hopes that these mapping data can be used to target vaccination campaigns more successfully with the aim of eradicating typhoid fever [9]. Based on this pattern recognition background and our 6-year experience of having methodologically and sequentially documented macroscopic and microscopic geometric patterns in malignant cancer tissues, we resolved to investigate the geomorphology patterns of 178 confirmed impact sites, taken from New Brunswick University's database (Canada), using Google Earth, Flash Earth, and Virtual Earth satellite platforms.

\section{Results}

\section{Statistical Analysis}

1) Megascopic GTCHC Complexes

We identified and documented components of crystal comet tail effect, helical, triangular, and hexagonal geochiral patterns in 126 (70\%) of 178 earth impact events analyzed (Table 1). These chiral patterns were found to be identical to those observed in cancer intercellular collisions (Figure 3).

We present nine of the most representative evidence of geochirality:

Table 1.

\begin{tabular}{ccc}
\hline Earth Impacts & GTCH Complexes & Percent \\
\hline 178 & 126 & $70.78 \%$ \\
\hline
\end{tabular}

Abbreviation: GTCHC. Geometric triangular chiral hexagonal like crystal complexes.
Bigach crater: $48^{\circ} 36^{\prime} 0^{\prime \prime} \mathrm{N}, 82^{\circ} 0^{\prime} 0^{\prime \prime} \mathrm{E}$. Satellite images show chain of fractal hexagonal pattern linked by comet tail sub collision hexagonal patterns that are structured on the basis of triangular chiral images (Figures 4 and 5).

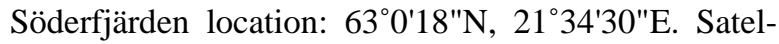
lite images show perfect hexagonal pattern assembly by triangular chiral images with fractal image and comet tail effect (Figures 6A and B).

Amelia Creek location: $20^{\circ} 51^{\prime} 0 " \mathrm{~S}, 134^{\circ} 53^{\prime} 0^{\prime \prime} \mathrm{E}$. Satellite images show fractal hexagonal patterns with comet tail structured based on triangular chiral images (Figures 6C and D).

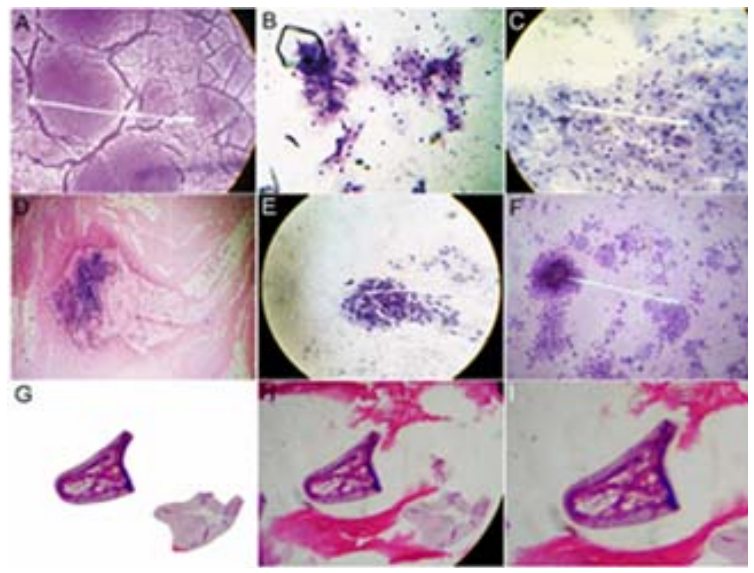

Figure 3. A-F Show microscopic sample of comet tail effect that generates an invariant hexagonal pattern on intercellular cancer collision event. With Papanicolaou staining (40×); G, H, I show triangular mirror image assembly in a case of skin cancer with hematoxylin and eosin staining (20×). Credit: Cancer Management and Research http://dx.doi.org/10.2147/CMAR.S25810.

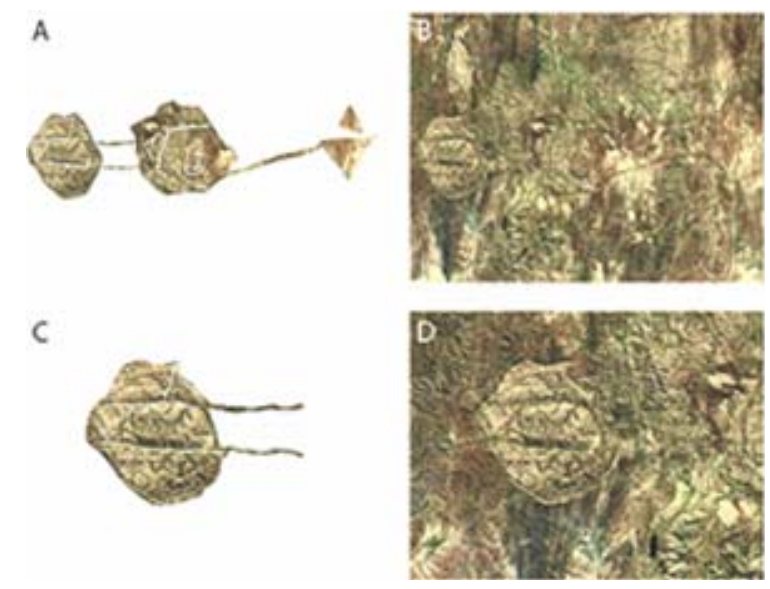

Figure 4. A show detachment image of $B$ that illustrates Bigach Crater $48^{\circ} 36^{\prime} 0 " N, 8^{\circ} 0^{\prime} 0$ "E. Satellite images revealing panoramic chain of fractal hexagonal patterns linked by comet tail effect, patterns are structured on the basis of triangular chiral images. $C$, show detachment image of $D$, that show close up the geometric complex. 

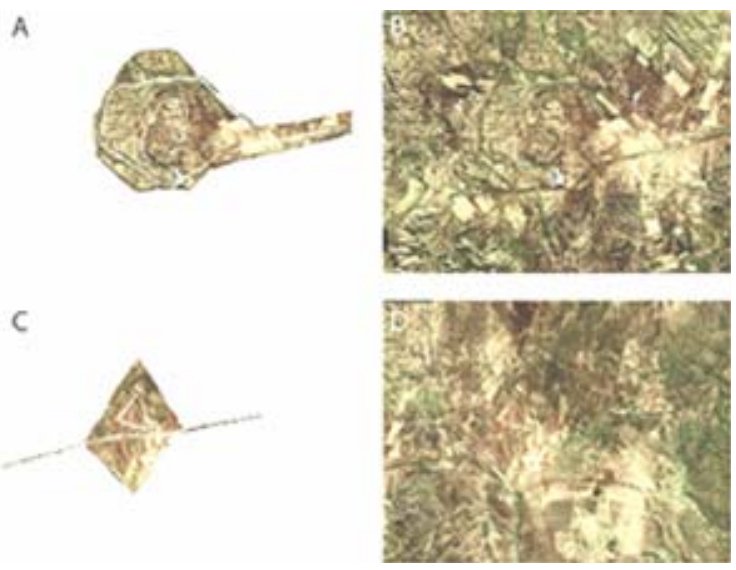

Figure 5. A, C show detachment image of $B$, and $D$, that show close up Bigach Crater geometric complex.

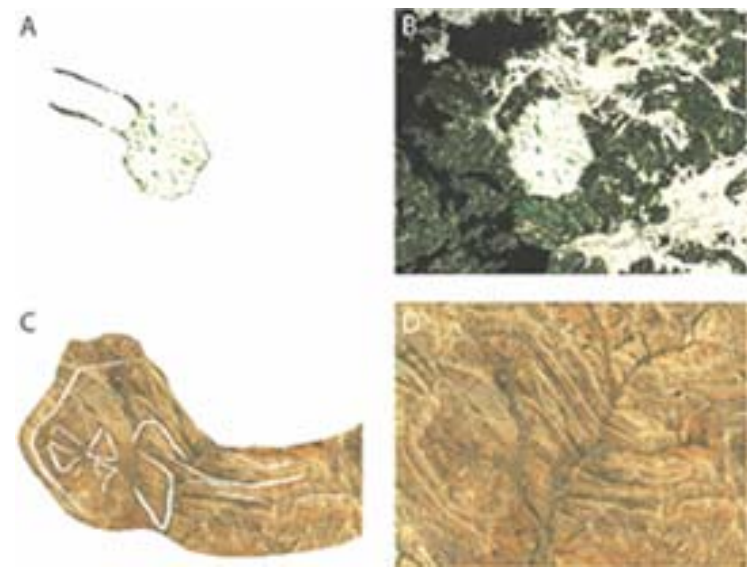

Figure 6. A show detachment image of $B$ that illustrates the Söderfjärden's crater location 63ㅇ' $18 " \mathrm{~N}, 21^{\circ} 34^{\prime} 30^{\prime \prime} 30^{\prime \prime E}$. Satellite images shows perfect hexagonal pattern assembly by comet tail effect. structuring in basis of triangular mirror images. $C$ show detachment image of $D$ that illustrates the Amelia Creek's crater location $20^{\circ} 51^{\prime} 0 " \mathrm{~S}, 1^{\circ} 4^{\circ} 53^{\prime} 0 " \mathrm{E}$. Satellite images show fractal hexagonal patterns with comet tail structured on the basis of triangular chiral images.

Aorounga, $19^{\circ} 6^{\prime} 0^{\prime \prime} \mathrm{N}, 19^{\circ} 15^{\prime} 0^{\prime \prime} \mathrm{E}$. Satellite images show fractal hexagonal patterns with comet tail structured based on triangular chiral images (Figures 7A and B).

Charlevoix crater: $47^{\circ} 32^{\prime} 0^{\prime \prime} \mathrm{N}, 70^{\circ} 18^{\prime} 0^{\prime \prime} \mathrm{W}$. Satellite images show attractor spin pair patterns in the opposite position mirror image in clockwise and anticlockwise orientation, linked by triangular geometric pattern (Figures 7C and D).

Henbury impact crater: $24^{\circ} 34^{\prime} 19^{\prime \prime S}, 133^{\circ} 8^{\prime} 54^{\prime \prime E}$. Satellite images show perfect visible hexagonal geometry with comet tail-like pattern identical to that observed at microscopic level in intercellular cancer collision event (Figures $8 \mathbf{A}$ and $\mathbf{B}$ ).

Zhamanshin in Kazakhstan: 48 $24^{\prime} 0^{\prime \prime} \mathrm{N}, 60^{\circ} 58^{\prime} 0^{\prime \prime} \mathrm{E}$. Satellite images show fractal hexagonal pattern with attractor interface organization structured by helicoidal band
A
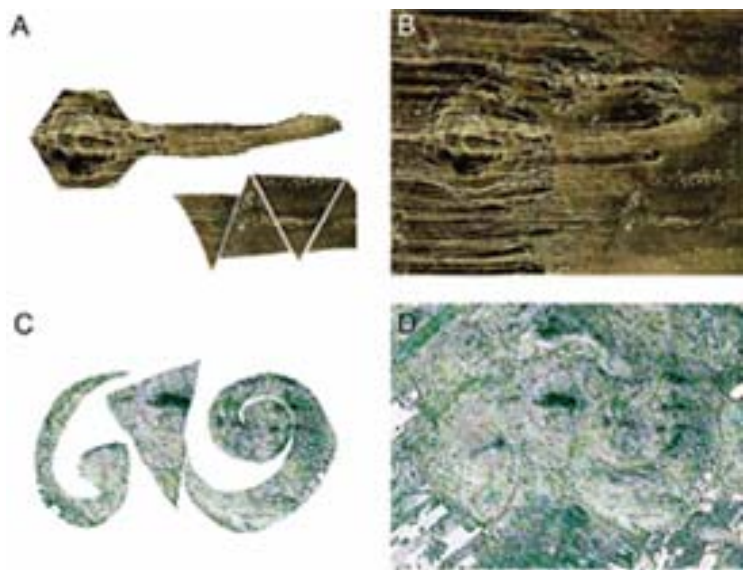

Figure 7. A show detachment image of $B$, that illustrates the Aorounga's Crater location $19^{\circ} 6^{\prime} 0^{\prime \prime} \mathrm{N}, 1^{\circ} 15^{\prime} 0^{\prime \prime E}$. Satellite images show fractal hexagonal patterns generate by comet tail and structured on the basis of triangular chiral images. $C$ show detachment image of $D$, that illustrates the Charlevoix's crater $47^{\circ} 32^{\prime} 0^{\prime \prime N}, 70^{\circ} 18^{\prime} 0 " W$. Satellite images show attractor spin pair patterns in opposite position mirror image in clockwise and anti-clockwise orientation linked by triangular geometric pattern.
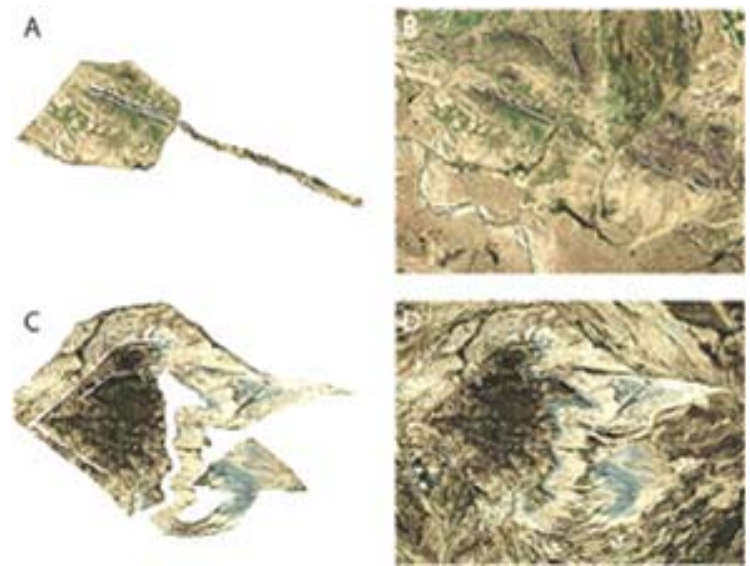

Figure 8. A show detachment image of $B$, that illustrates the Henbury's crater $24^{\circ} 34^{\prime} 19^{\prime \prime}$, $133^{\circ} 8$ '54"E. Satellite image show perfect visible hexagonal geometry with comet tail like pattern identical to those observed at microscopic level in cancer intercellular collision event. $C$, show detachment image of $D$, that illustrates the Zhamanshin impact event in Kazakhstan $48^{\circ} 24^{\prime} 0 " \mathrm{~N}, 6^{\circ} 58^{\prime} 0^{\prime \prime E}$. Satellite images show fractal hexagonal pattern with attractor interface organization structured by helicoidal band that link two triangular chiral images, perfect hyperorder domain.

that links two triangular mirror images' perfect hyperorder domain (Figures 8C and D).

Manicouagan impact crater: $51^{\circ} 23^{\prime} 0^{\prime \prime} \mathrm{N}, 68^{\circ} 42^{\prime} 0^{\prime \prime} \mathrm{W}$. NASA satellite image shows clear blue stripe line band that forms geospatial relation between the blue stripe line and the magnetic abnormalities over the surface of the terrain that structures triangular mirror images at each side in their trajectory. This is an absolutely manifested 
direct visible proof of the strong energy released from the bolide's fast speed in the collision event (Figures 9A and B).

Manicouagan impact crater: $51^{\circ} 23^{\prime} 0^{\prime \prime} \mathrm{N}, 68^{\circ} 42^{\prime} 0^{\prime \prime} \mathrm{W}$. Satellite image shows triangular white-black chiral images in the center of the impact area (Figures $9 \mathbf{C}$ and $\mathbf{D}$ ).

2) Visualization of Novel Earth Impact Event

During the analysis of the 178 earth impacts obtained from the database of New Brunswick University through satellite tools, such as Google Earth, Yahoo Maps Virtual, and Flash Earth, we identified and documented a novel earth impact event that has generated crystal comet effect tail with geometric triangular chiral hexagonal hyperorder domain with the expression of strong MF, creating a visible infrared green stripe line spectra detected by satellite sensors but probably invisible to the naked eye (Figure 10).

3) Morphology

Satellite images show circular structure, depression with raised rims and/or central uplifts, and multi-ring structures with radial circumference of $3.30 \mathrm{~km}$. With anomalous geological character or geophysical expression, its appearance is similar to a complex crater. Pseudotachy lite associated with the impact structure may be formed in radial and concentric fault systems, which helps to define the megascopic structure of the crater on the earth, recognizing the impact center or the bull's eye.

4) Geologic Evidence

The following were observed to be regularly found inside and around the impact structure: strong deformations,
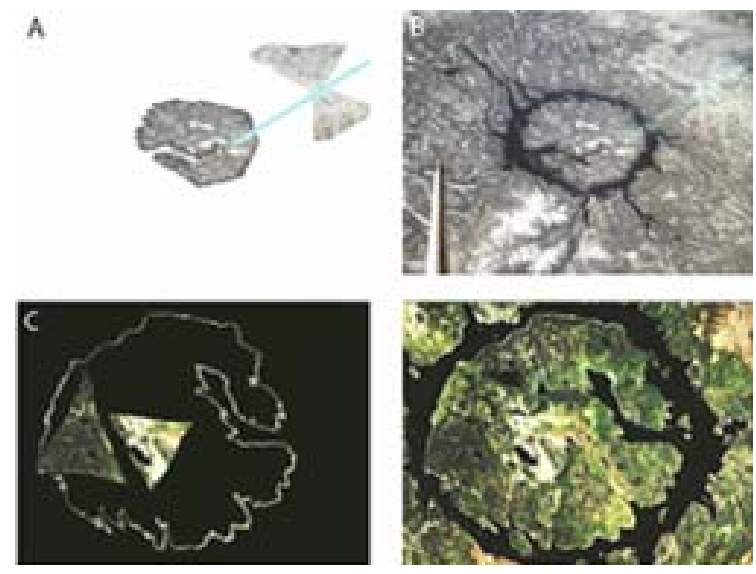

Figure 9. A show detachment image of $B$, that illustrates the Manicouagan's crater $51^{\circ} 23^{\prime} 0 " \mathrm{~N}, 6^{\circ} 42^{\prime} 0$ "W. NASA satellite image shows clear blue stripe line that cause geospatial interaction between the stripe line and the magnetic abnormalities over the surface of the terrain that structure triangular mirror images at each side in their trajectory. Direct visible proof of the strong energy release by the bolide's speed in collision event. $C$, show detachment image of $D$, which illustrates other angle of Manicouagan's crater. Satellite image show in the center of impact triangular white-black mirror images.

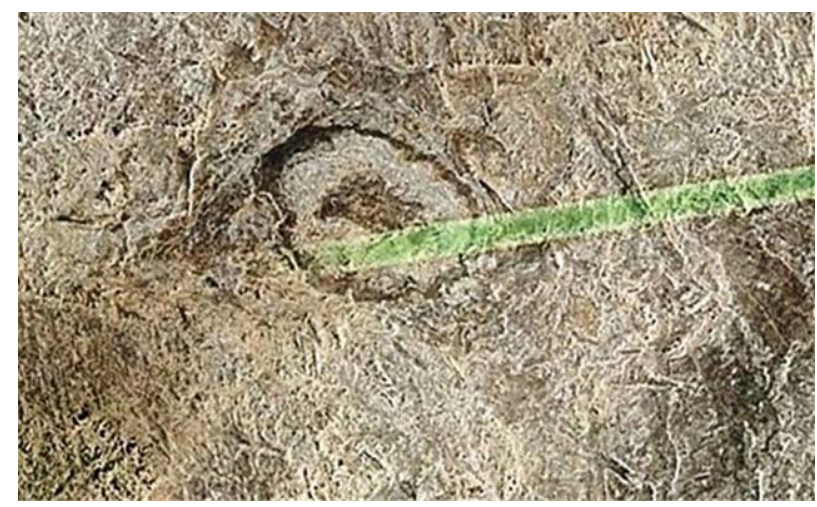

Figure 10. Show identification of novel megascopic collision event, Satellite image show close up of green stripe line 128 km ending in convergence with hexagonal geomorphic and circular structure bull's-eye.

folding, faulting, fracturing, breccias and dike breccias, mega breccias, high-pressure/short-term deformations of clash in a soft matrix, mapped rocks showing the effects of convincing metamorphic shock in places near the impact, planar fractures visible to geomorphic expression of strong MFs, and magnetic hyper-order.

The observable comet effect tail represents the bolides' fast trajectory, similar to giant lightning strikes. Particles are accelerated along the open field lines near the friction with the environment atmosphere and leave an observable track expressed by a green stripe oblique linear spectra trajectory, perfectly visible in different satellite platforms but probably invisible to the naked eye. The observable expression of strong magnetic force is generated on the ground surface in terms of the following: Helical pattern (Figure 11) hexagonal geometry patterns (Figure 12A), and geology well defined mirror images (Figure 12 B), indicating that the stripe is not a pixel error of the photography and that this stripe is real.

Perfectly green stripe line spectra converge with the hexagonal pattern displayed on the surface to the terrain. The geospatial relation between the green stripe line and the magnetic abnormalities over the surface of the terrain is a direct visible proof of the strong energy release from the bolides' speed energy in the collision event. Accordingly, the geometric patterns in the terrain appear to be younger than the green stripe line, indicating that the collision surface is still evolving in a surprising way. Comparative satellite sensory infrared images analysis documenting the green electromagnetic stripe line along side different zones of their trajectory coincides perfectly with the satellite no sensory infrared images, illustrating how real its the geo-line documented in situ. (Figures 12-15).

We were able to recognize that the geologic material that blasted out of the crater may form eject blankets around the original central cavity. For large impact events, 

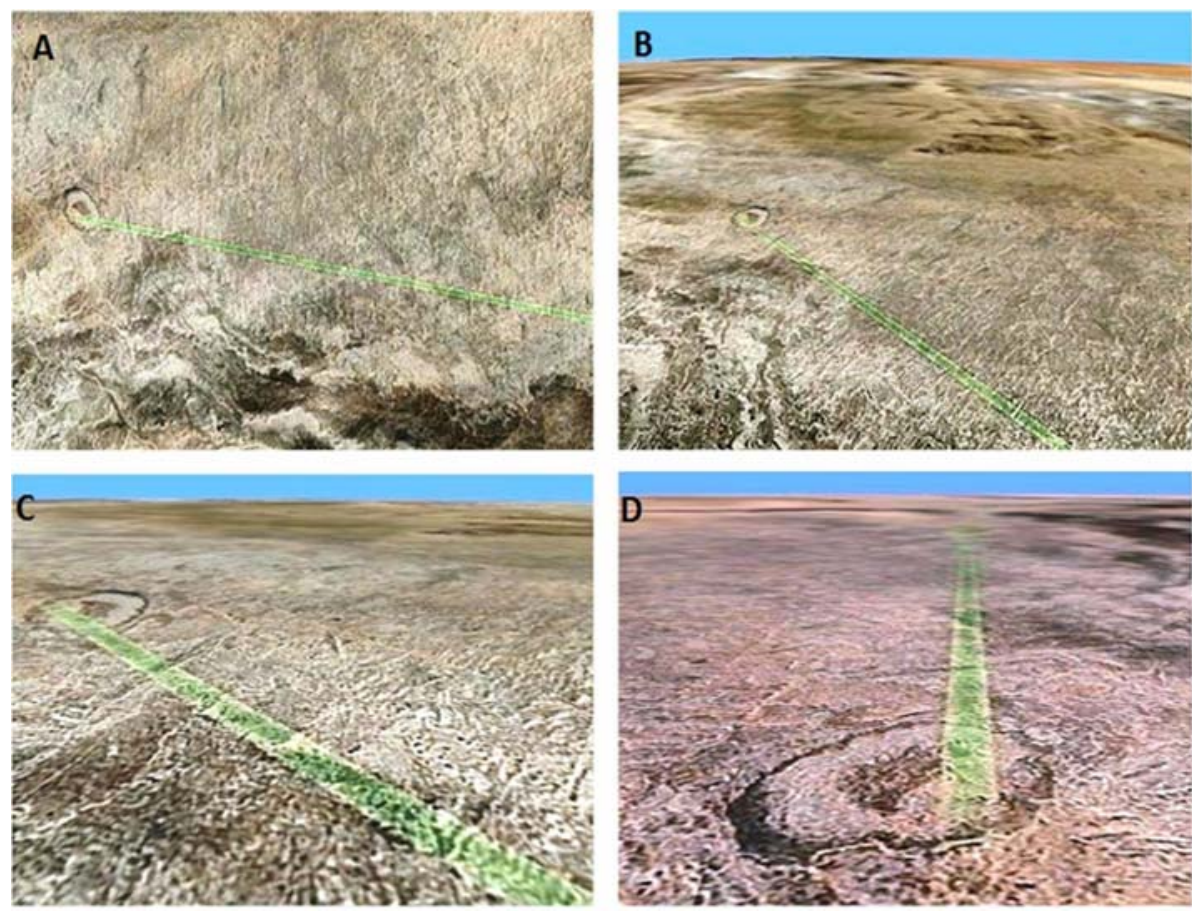

Figure 11. A-D Satellite image show the geospatial effect interaction of the green stripe line over the surface terrain in terms of generation of helicoidally pattern throughout the trajectory of the line.
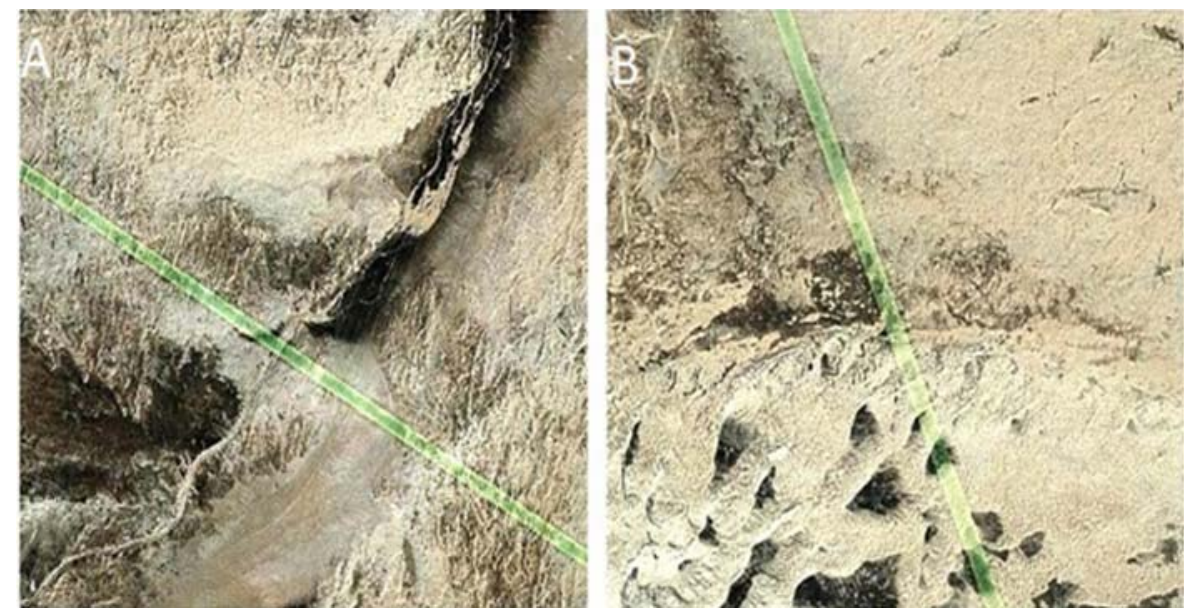

Figure 12. A Satellite image show the interaction effect that generate the green stripe line magnetic field in the terrain surface structuring invariant hexagonal pattern. B Satellite image show geology well defined mirror images at each side of the green stripe line trajectory.

the eject blankets could be distributed globally. Impact melt sheets are recognized by careful mapping and rock sampling. In the area of the impact interaction, satellite images show an impressive beautiful pair of triangular geo-chiral structures located $61.05 \mathrm{~km}$ north from the primary impact. This monumental structure has not been revealed earlier. The principal structure with a height of $550 \mathrm{~m}$ and width of $645 \mathrm{~m}$, and has a pyramid triangular shape with elevated plateau (Figure 16); their adjacent chiral counterpart has the same dimensions but inverted chiral position with apparent central excavation filled with water process (Figures 17A-D).

5) Megascopic Eclectic Rock Art Hieroglyph

The satellite image shows, in the area of the impact interaction, $60 \mathrm{~km}$ south of the main crater, rock art hieroglyph geomorphic pieces ready to be deciphered, represented by three megascopic perfectly aligned pyramidal structures: the minor with comet tail in the same cleavage plane, geochiral triangular mirror images linked by helical pattern, and the presence of embryoid body inside one of them (Figures 18A-D). In the adjacent area, we could identify other monumental triangular mirror 

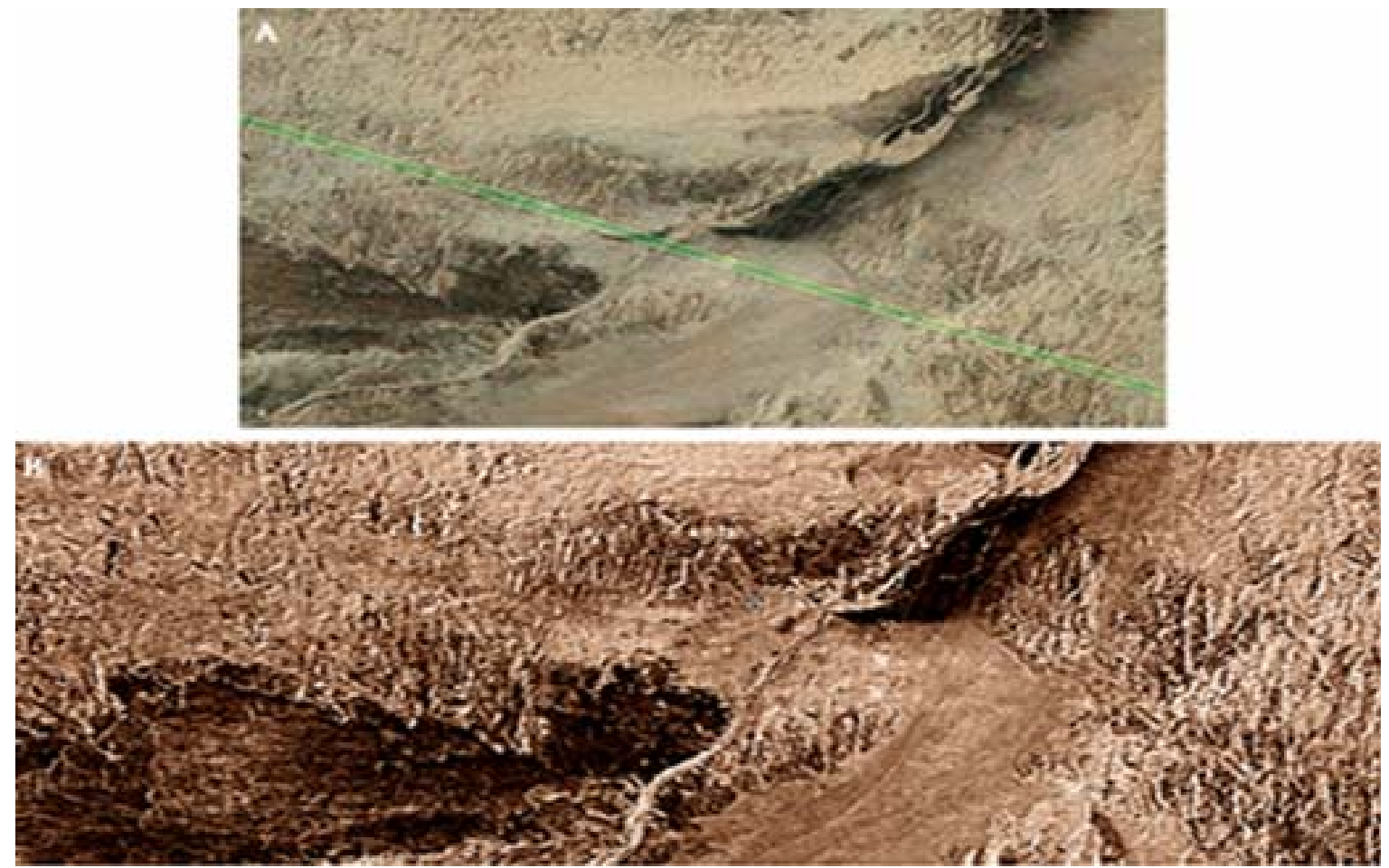

Figure 13. We verify that the geo-line image features are not artifact for two principal reasons: Comparative satellite sensory infrared images analysis documenting the green electromagnetic stripe line coincides perfectly with the satellite no sensory infrared images, of the stripe trajectory illustrating how real its the geo-line.
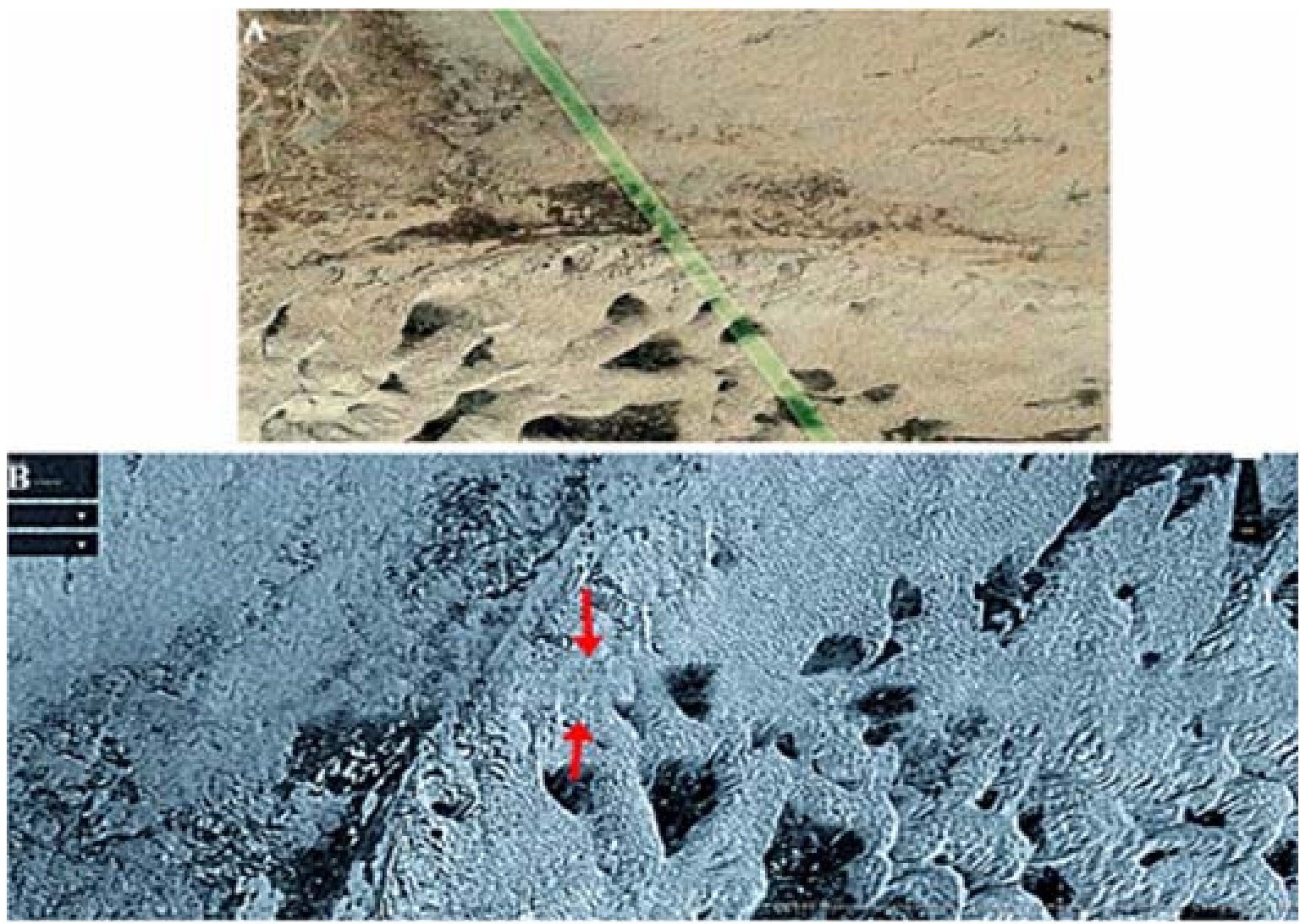

Figure 14. The electromagnetic field of the green line generates mirror images on each side of its trajectory. 


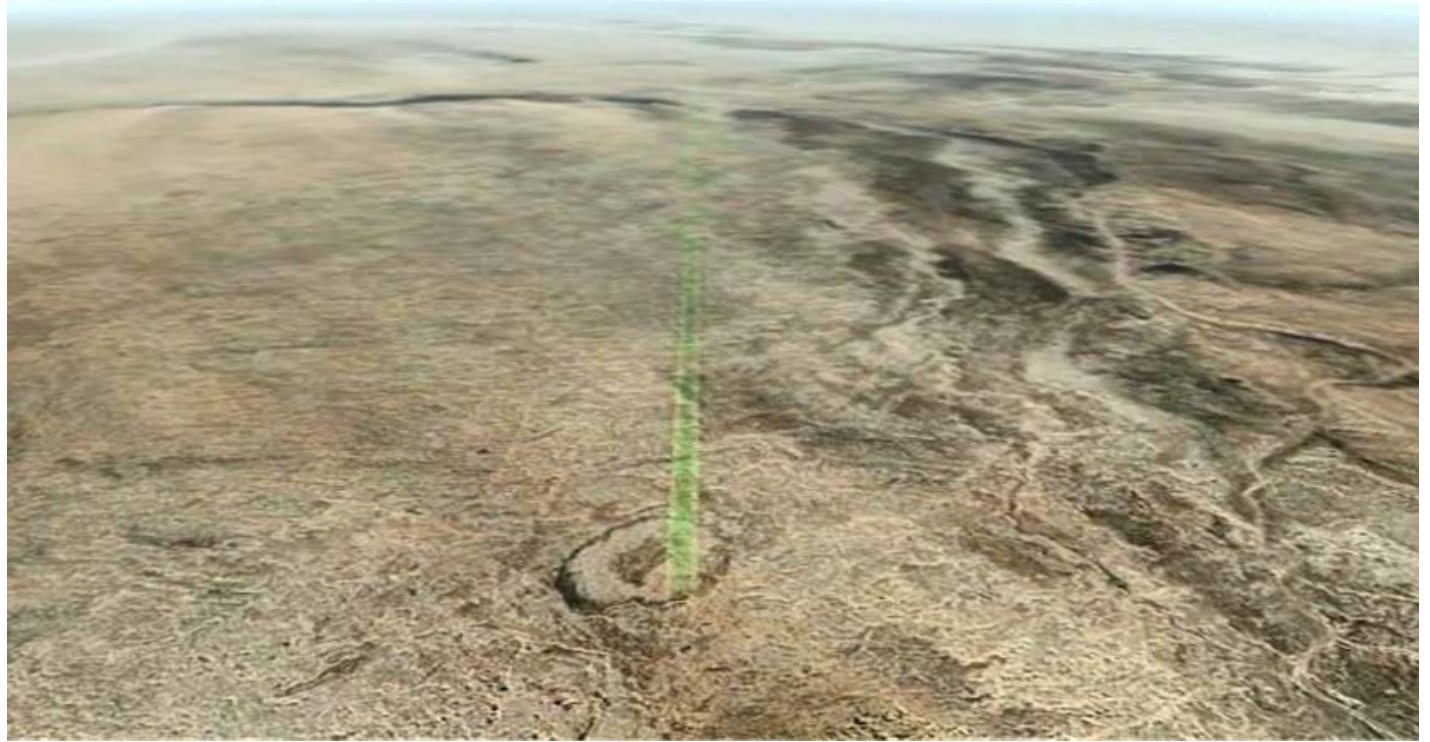

Figure 15. Satellite sensory infrared images analysis documenting the green electromagnetic stripe line.

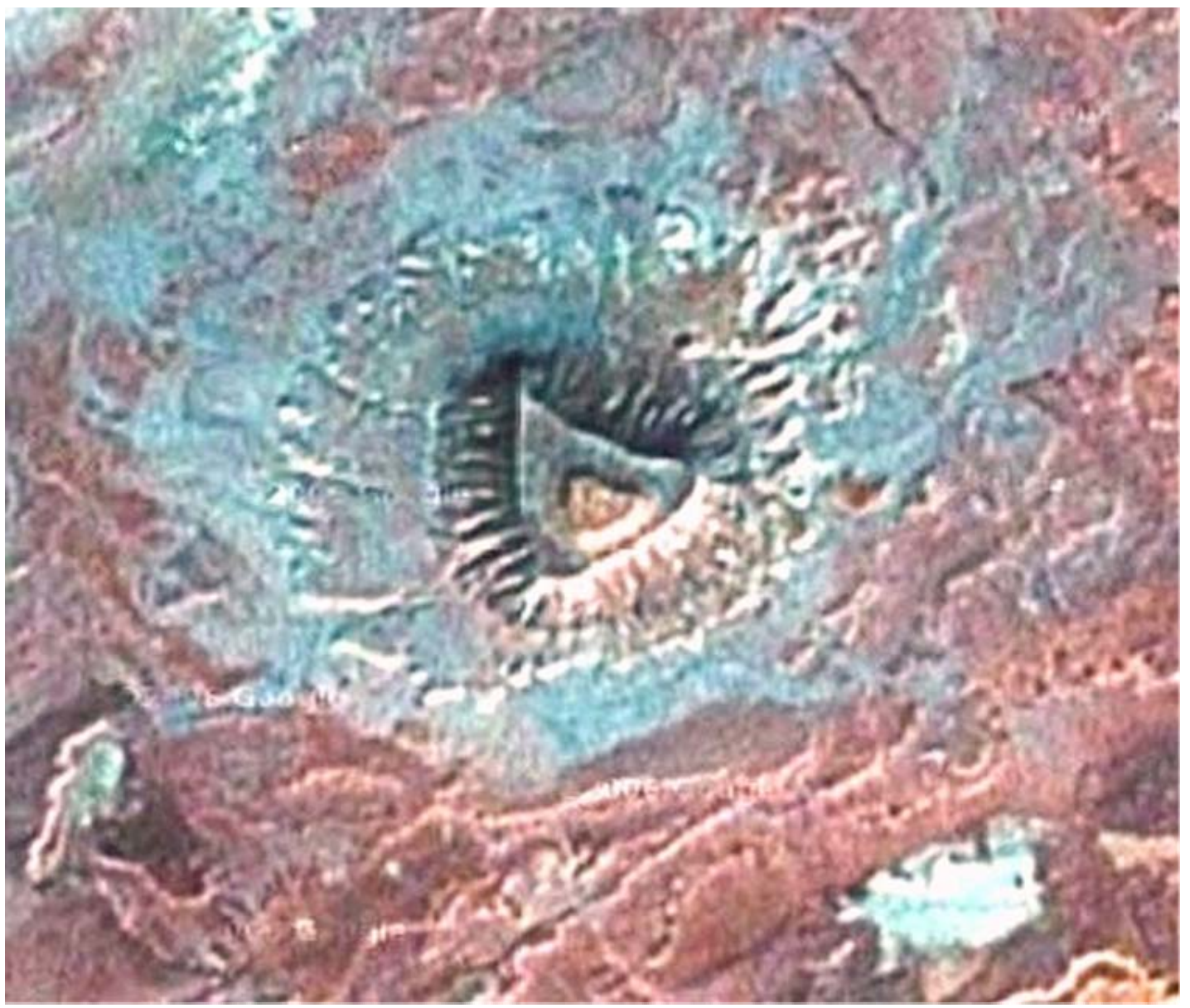

Figure 16. Satellite images show in the area of the impact an impressive beauty pair triangular geo chiral structures located $61.05 \mathrm{Km}$ north from the primary impact. This monumental structure has not been revealed before. The principal structure is "without parallel", with a height of 550 meters and a width of 645 meters; has a pyramid triangular shape with elevated plateau. 

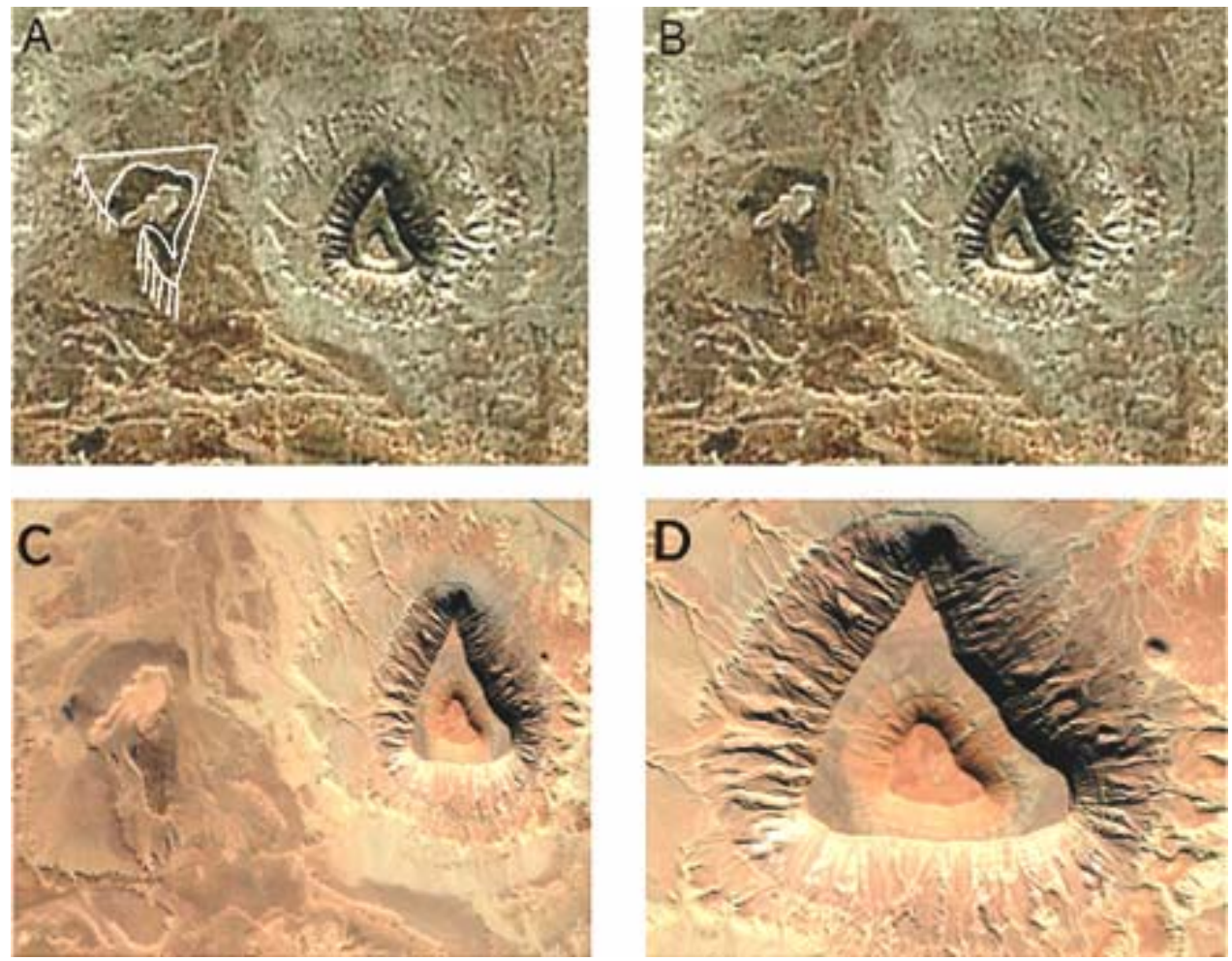

Figure 17. A, B Satellite images show pyramidal triangular self-assembly geologic structure with their chiral counterpart adjacent side by side. Same dimensions but in inverted chiral position with apparent central excavation filled with water process. C, D, aerial photography close up image.

A
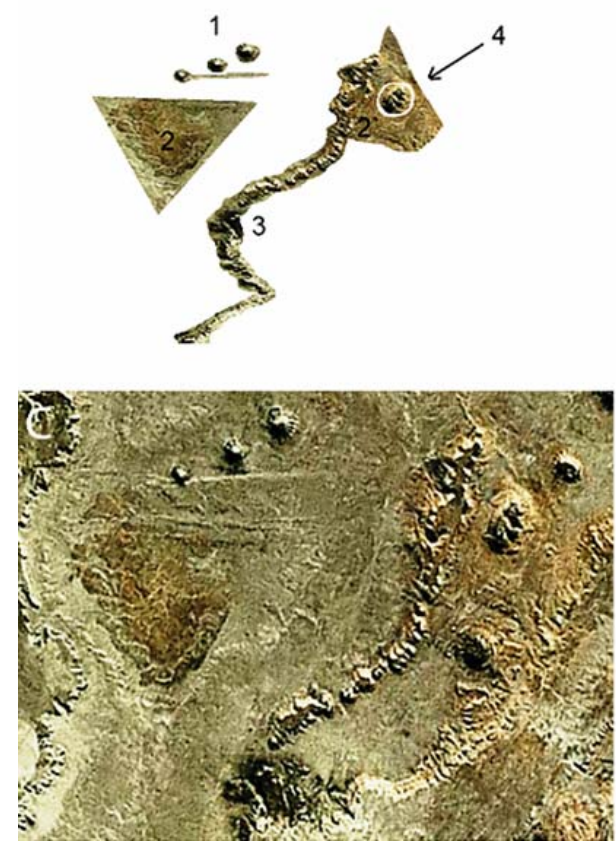
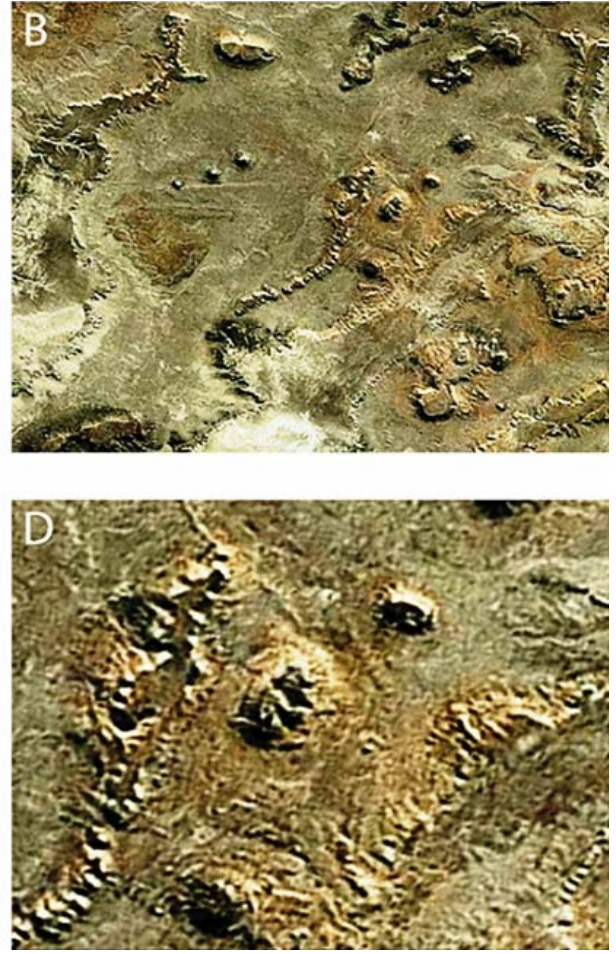

Figure 18. Megascopic eclectic rock art hieroglyph Satellite images A-D, show the area of the impact interaction; but located $60 \mathrm{~km}$ south of the main crater, rock art hieroglyph geomorphic pieces ready to be deciphered, represented by three megascopic perfect aligned pyramidal structures the minor with comet tail, in the same cleavage plane; geo-chiral triangular mirror images linked by helical pattern; with the presence of embryoid body on the surface. 
rocks (Figures 19A-D) and biosignature template platform of primitive head tail organization. Furthermore, we could also identify embryoid body and human-like shapes sculpted as giant fossils in the rocks around the impact area (Figures 20-22). Finally we identify like dinosaur structure located 110 kilometers from the area of impact (Figure 23).

\section{Discussion}

What is the meaning of the intercellular cancer collisions?

Cancer is, by definition, the uncontrolled growth of autonomous cells that eventually destroys adjacent tissues and generates architectural disorder, and also shows how a cell population undergoing density-dependent growth can lead to a chaotic dynamic biological system, moment at which thousands of malignant cellular membranes embark on a gradually increasing collision process as these cells multiply in disorder, at this point collider partners-crystal particles that spin in opposite directions in synchronic and simultaneous movement may point "left" or "right". This rotation generates powerful magnetic field lines. The electron particles are aligned in their domain and the flux in the magnetic lines are distributed in the extracellular matrix along the collagen and vascular components.
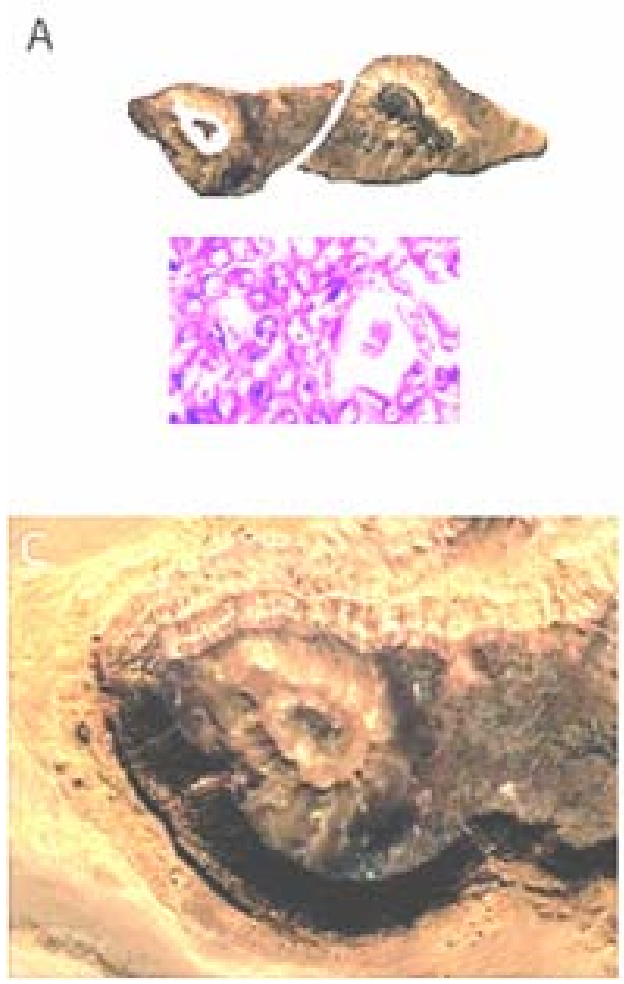

Because electrons flow spirally through a conductor (collagen type I), these magnetic lines move with the spiral. Pair production, inherent pair development and dependence, induces the flux to flow in the opposite direction. In this manner, particles expand or go away, and others come closer or are contracted from a fixed point. This entangled phenomena produce dipole behavior in terms of metabolic proliferation and apoptosis status, a process that is revealed in multiple outbursts of biological collisions in malignant tumors. This complex is fractal, always evolving into more complex geometric hexagonal structures over time-space intervals. It is a predictable and reproducible dynamic system. It is identified on systems in states of disorder regardless of the type of system involved. It has an invariant morphology with sets of triangular mirror images in opposite positions and spiral-patterned visible attractors. It is an irreducible geometric matrix.

Under this novel observation cancer incorporates a real visualization world with a great surprising finding in biology, physics, and geology. This visualization platform literally allows us to see what would otherwise remain completely invisible. In a brief glance, we could recognize patterns or identify suitable features in noise data—something that is difficult or impossible to achieve with more traditional data analysis.
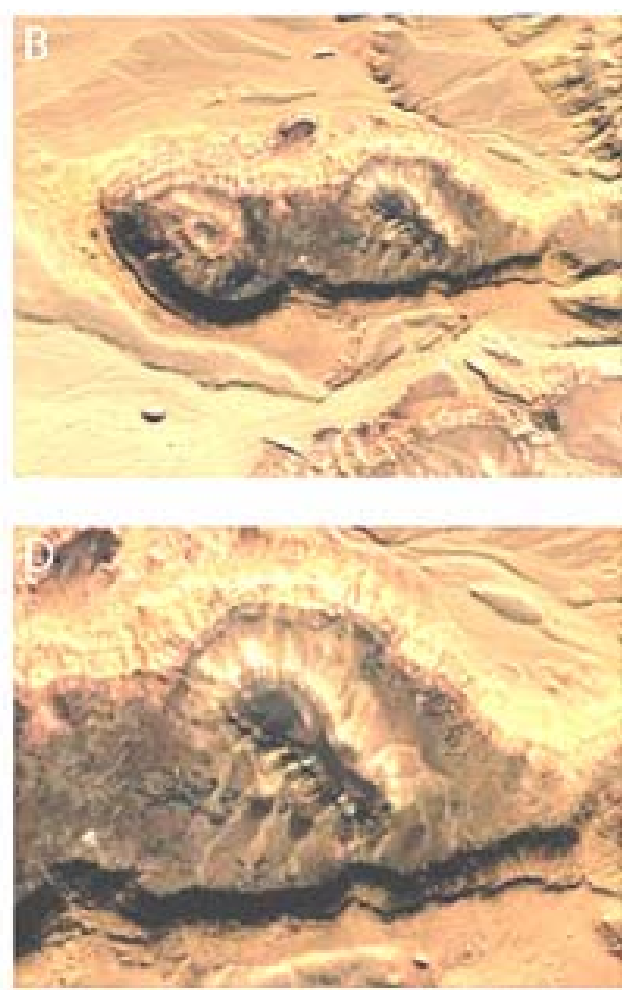

Figure 19. Aerial photography. A, show detachment and comparative microscopic GTCHC analisis image with B-D, geologic monumental triangular mirror image structures, associate with chiral white-black, head-tail geomorphic patterns embedded on the rock surface. 

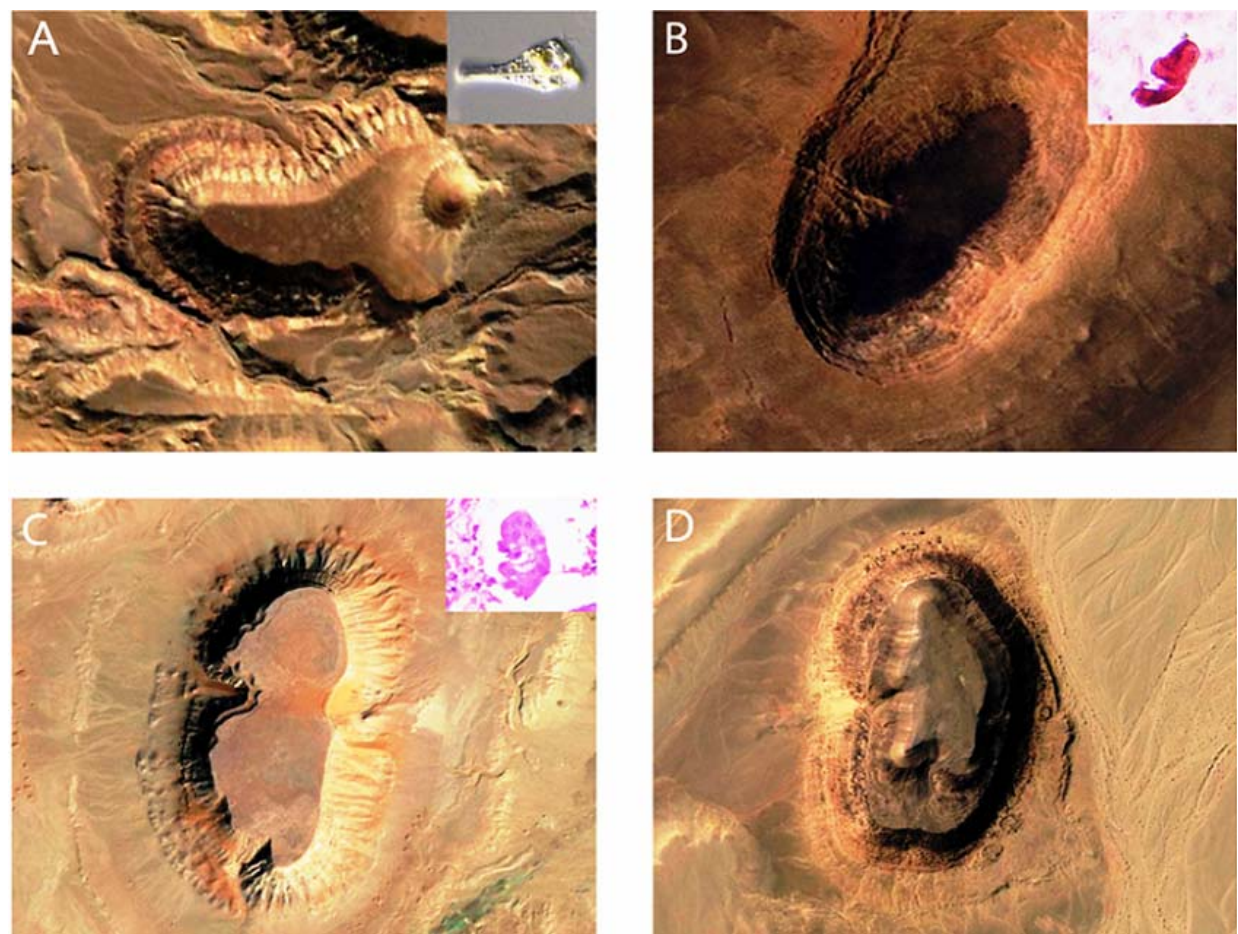

Figure 20. Aerial photography show in A, comparative image analysis between Collodictyon ${ }^{*}$ an ancient lineage in the tree of eukaryotes and primitive structure head tail organization like fossil embedded in the rock. B, C, Comparative image analysis between embryoid body self-assembly geologic structures with embryoid body self-assembly observed in cancer intercellular collisions. D, Human being shaped like giant fossil embedded in the rock. ${ }^{*}$ Credit: Kamran Shalchian-Tabrizi, of the University of Oslo, Norway.
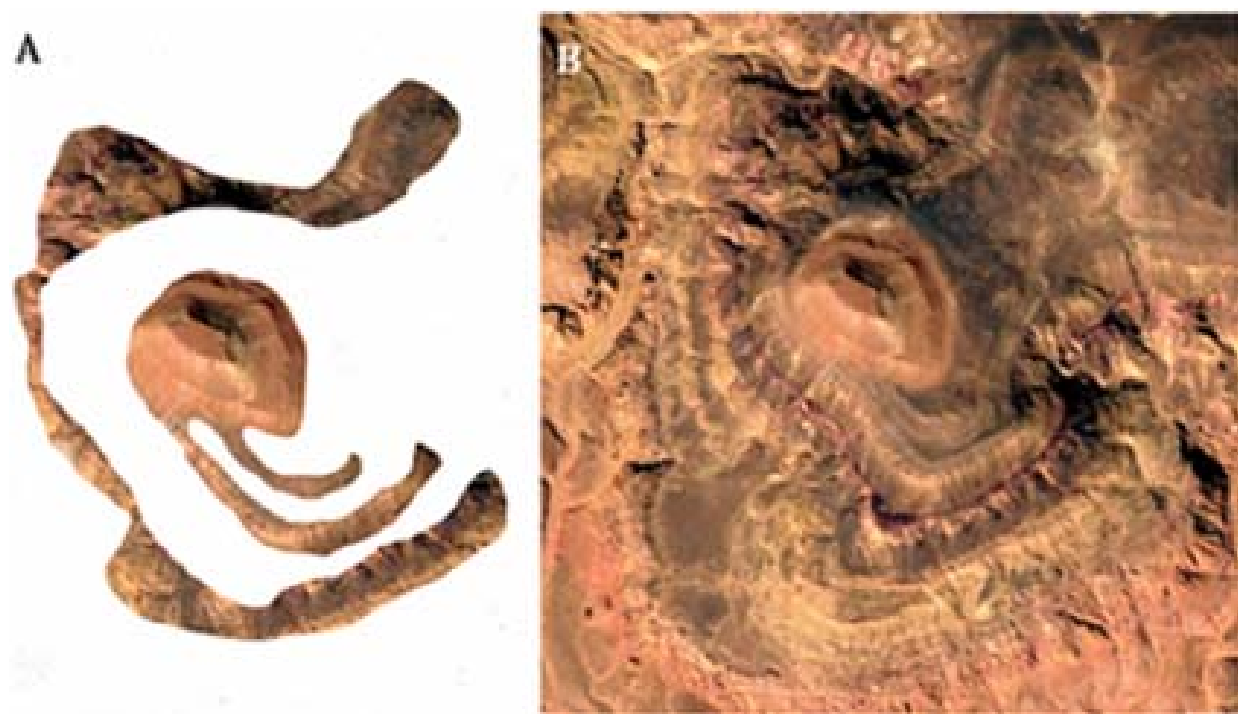

Figure 21. A, show detachment image of B, illustrating embryoid body geomorphic pattern.

Can be very difficult to understand the relationship between the cancer and earth impact event, to facilitate an approach to this question, we should raise this concern in context of visual perception: How we can explain the identification of geometric similar patterns in dissimilar systems? We are probably confronted with irreducible geometric matrix, noble structures that remain unchanged at the microscopic, macroscopic, megascopic levels during transit. Thus, independent of the type of collision event, collisions generate geometry in a space-time interval. In the same way, in any given place, geometry has existed previously in the interaction area as a background of a collision event.

Cancer GTCHC complex natural model predict and 

geomorphic shape pattern.

\section{A}

reproduce realistic values in scaling relationships, tested with the identification and localization of geology hidden self-assembly geometric structures related to earth impact events.
A
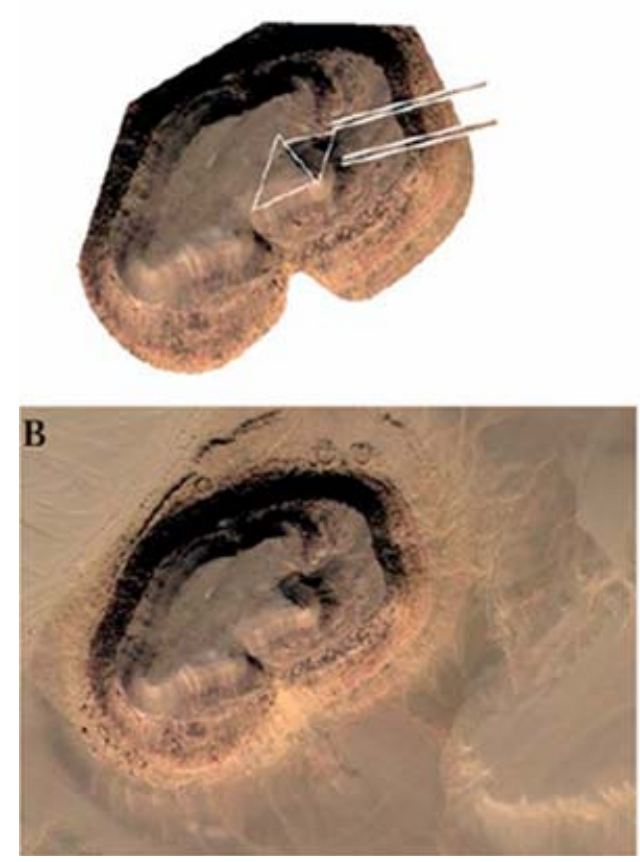

Figure 22. Aerial photography. A, show detachment of B, horizontal position of image C, illustrating Rock Human being
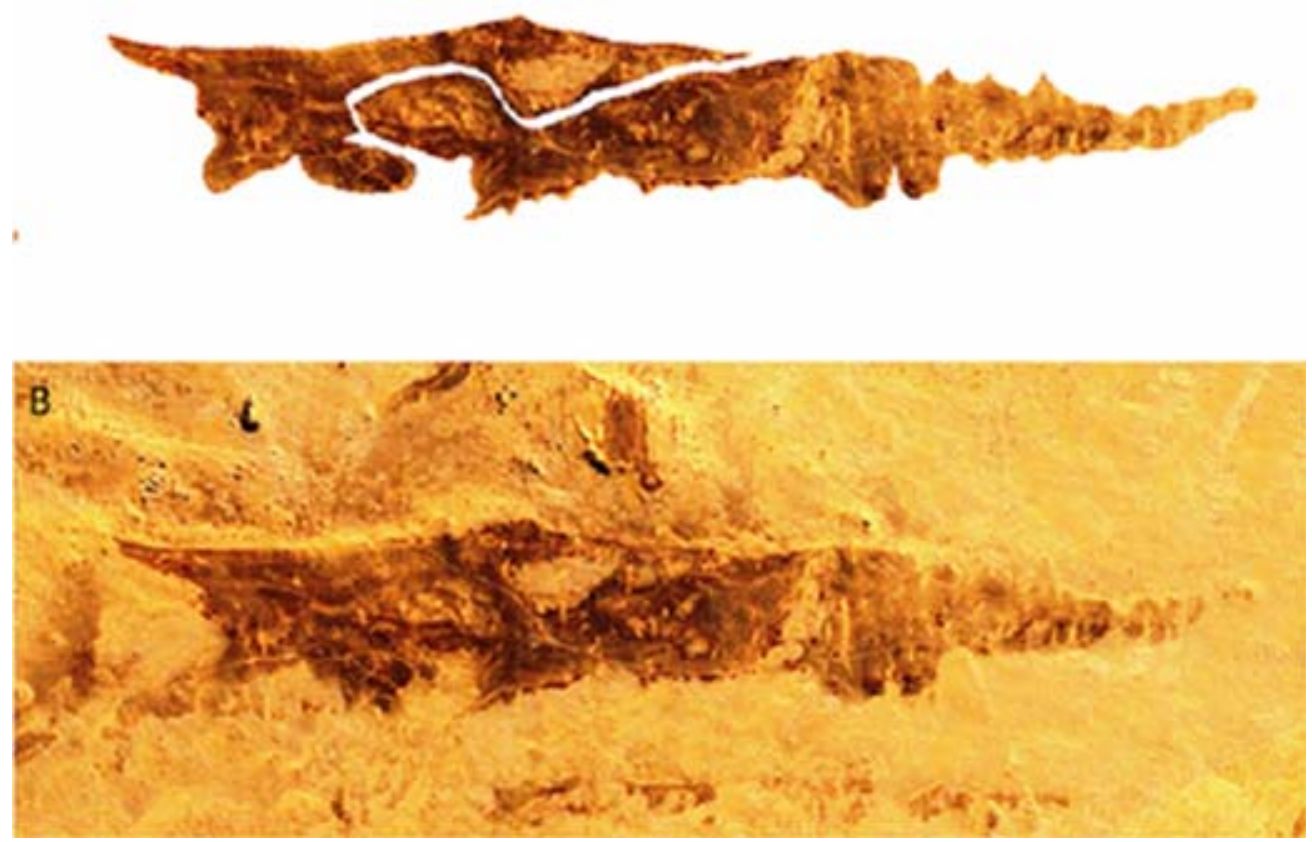

Figure 23. A, show detachment image of B, illustrating like dinosaur geomorphic structure.

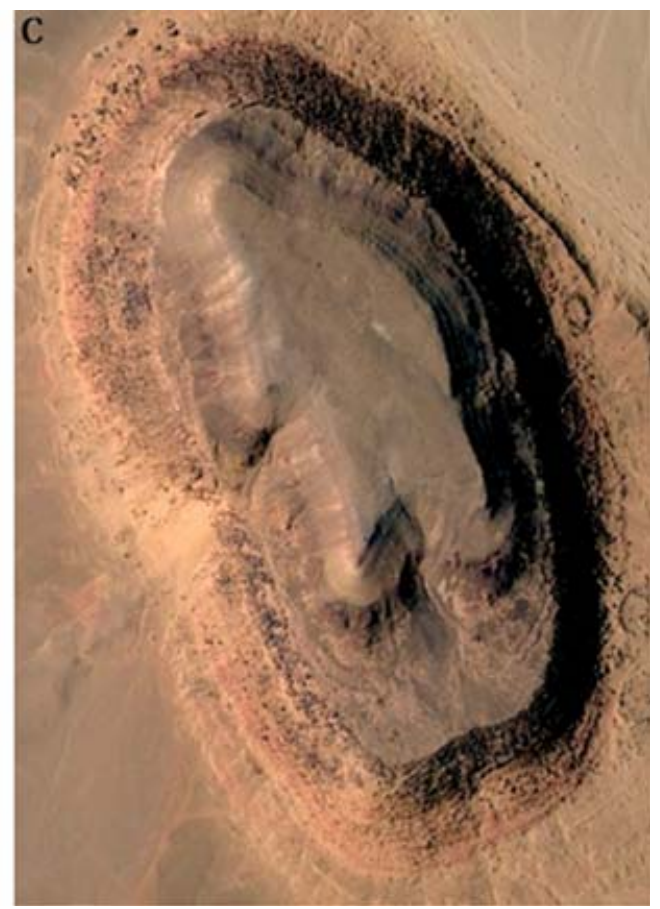

From theory to practice our data observations in this irreducible geometric matrix allowed us to identify, in geology, real measurable green infrared-electromagnetic stripe line in interface with hexagonal geomorphic pat- 
tern, triangular chiral pyramidal rock structures, template platform to biosignature characterization of ancestral primitive polar head-tail organization, and embryoid and human-like shape pattern embedded as giant fossils in rocks that have never been seen before. Cancer is narrating us a story of how probably life started on our planet.

Following the exploration of UCLA chemistry and physics research group on how and why chirality arises, and their determination of how chirality has a physical origin, our observations in cancer chaos systems has become more relevant. In physics, we are faced with invariant standard pattern: triangular chiral self-assembly structures. The biology of cancer collisions and geomorphic documented patterns of comparative image analysis are self-explanatory, and the observable similarity is absolutely impressive. From these images that act as evidence, we can state with absolute consensus that collision events generate in the spectrum of space-time biosignature hyper-order domain states at all scales, which corroborates the idea that the dynamic geometric order occurs through an MF activity On the basis of this hierarchical order, we were able to recognize order in the existence of different kinds of complex systems that, under difficult levels of complexity, are organized in the whole domain of similarities by self-assembly.

We were able to observe the invariant geometric similarity patterns as the manifestation of a primitive underlying process - entropy. Thus, we can no longer consider rocks, lands, animals, and human beings as separate entities. Geologic forces establishing the conditions for life on earth and characteristic patterns are repeatedly found in descending scales, and hence, their parts on any scale must be similar in relation to the whole. Experimentally, researchers have observed MF-induced charge-stripe order in high-temperature superconductor [10].

It would have been practically impossible to achieve localization of this place and their specific geographic coordinates if we do not possess previous knowledge on substrate morphology to understand and decode the encrypted information hidden in this fractal geometry. Thus, this fact represents the most unambiguous link between the microscopic and macroscopic worlds where geology, physics, and biology converge into a merged entity.

The visualization of this geometry and biosignature patterns in collision impact areas suggests that those chiral enantiomer primordial molecules are self-assembled in this interaction zones. Meteorites hold a record of the chemicals that existed in the early solar system and that may have been a crucial source of the organic compounds that gave rise to life on earth [11-17]. Since the 1960s, scientists have been trying to find proof that nucleo bases, the building blocks of our genetic material, came to earth on meteorites. A new research, published in the Proceedings of the National Academy of Sciences, indicates that certain nucleo bases had reached the earth from extraterrestrial sources through certain meteorites and in greater diversity and quantity than previously thought. Extensive research has shown that amino acids, which string together to form proteins, exist in space and had arrived on our planet piggybacked on a type of organic-rich meteorite called carbonaceous chondrites. [18] However, it has been difficult to similarly prove that the nucleo bases found on meteorite samples are not due to contamination from sources on the earth.

Life probably was created and sustained at the very edge of chaos. In consequence the earth impact zones, far from having generated destruction in the past, could be, based on our criteria, the most plausible nesting place for the origin of life in our planet; if form is a function, then it is conceivable that the hyper-order domains in theory could be portals hidden in the Earth's MFs, where tons of high-energy particles may flow through space turbulence and create heavenly zones of geometry order.

Recently understanding how space turbulence works, researcher conducts supercomputer simulations to learn impacts on earth's magnetic field, providing the first strong evidence for the ion entropy in colliding plasmas in an electromagnetic turbulence simulation [19]. Comparison between the predicted computer wave magnetic patterns behind these collisional plasmas with the geomorphic structures documented by us, shows great similarity and reproduce the collsionally enhanced damping rates of linear waves observed in plasma collision experiments. This fact validates and explains our observational approaches.

In conclusion electromagnetic field released in collision-impact events generate in the interphase matrix fractal scalable invariant irreducible geometric triangular chiral hexagonal structures associated with biosignature patterns. Geometric order could potentially be life-bearing.

We must consider the history of living beings as entities of geobiologic co-evolution. The laws of biology and geology can finally be redirected to the laws of physics. Specifically, MFs create a new kind of classification based on these fractal structural similarities of the relationship. Further interdisciplinary collaboration must be carried out to study these geometric self-assembly geological structures, ancient sediments and rocks that could provide insights into antecedents of life.

\section{Acknowledgements}

The authors thank Dr. Juan Carlos Triana and Dr. Jorge Ovidio Cruz, executive directors of the Hospital Department of Villavicencio and Hospital Department of Granada, for their financial support of this research as well as the medical students Mauricio Felipe Murillo and Jen- 
nifer Catherine Camacho for the picture work and panel arrangement.

\section{REFERENCES}

[1] J. Diaz, N. Jaramillo and M. Murillo, "Geometric Triangular Chiral Hexagon Crystal-Like Complexes Organization in Pathological Tissues Biological Collision Order," PLoS One, Vol. 2, No. 12, Article ID: e1282. http://www.plosone.org/article/info:doi/10.1371/journal.p one.0001282

[2] J. Diaz and M. Murillo, "Framework of Collagen Type I Vasoactive Vessels Structuring Invariant Geometric Attractor in Cancer Tissues: Insight into Biological Magnetic fields,” PLoS One, Vol. 4, No. 2, Article ID: e4506. doi:10.1371/journal.pone.0004506

[3] J. Diaz, M. Murillo and A. Barrero, "Intercellular Cancer Collisions Generate an Ejected Crystal Comet Tail Effect with Fractal Interface Embryoid Body Reassembly Transformation," Cancer Management and Research, Vol. 3, 2011, pp. 143-155.

http://www.dovepress.com/intercellular-cancer-collisionsgenerate-an-ejected-crystal

[4] J. Diaz and M. Murillo, "Phenotype Characterization of Embryoid Body Structures Generated by a Crystal Comet Effect Tail in an Intercellular Cancer Collision Scenario,” Cancer Management and Research, Vol. 4, 2012, pp. 921.

[5] K. Zhao, R. Bruinsma and T. Mason, "Local Chiral Symmetry Breaking in Triatic Liquid Crystals," Nature Communications, Vol. 3, 2012, Article ID: 801.

[6] L. Kugland, D. Ryutov, P. Chang, R. Drake, et al, "SelfOrganized Electromagnetic Field Structures in LaserProduced Counter-Streaming Plasmas," Nature Physics, Vol. 8, 2012, pp. 809-812. doi:10.1038/nphys2434

[7] A. Hickman, "Big Impact with New Meteorite Crater Discovers in Prospect,” Western Australia's International Resources Development magazine, 2008. http://www.enotes.com/topic/Hickman_Crater

[8] L. Folco, M. Di Martino, A. El Barkooky, M. D’Orazio, et al, "The Kamil Crater in Egypt," Science, Vol. 329, No 5993, 2010, pp. 804-807. doi:10.1126/science.1190990

[9] K. E. Holt, J. Parkhill, C. Mazzoni, et al, "High-Throughput Sequencing Provides Insights into Genome Variation and Evolution in Salmonella Typhi," Nature Genetics,
Vol. 40, No. 8, 2008, pp. 987-993.

doi:10.1038/ng.195

[10] T. Wu, H. Mayaffre, S. Krämer, et al. "MagneticField-Induced Charge-Stripe Order in the High-Temperature Super Conductor YBa2Cu3Oy,” Nature, Vol. 477, No. 7363, 2011, pp. 191-194. doi:10.1038/nature 10345

[11] R. Gilbert, "Lie Groups, Physics, and Geometry,” Cambridge University Press, New York, 2001.

[12] M. H. Engel, S. A. Macko, "Isotopic Evidence for Extraterrestrial Non-Racemic Amino Acids in the Murchison Meteorite,” Nature, Vol. 389, No. 6648, 1997, pp. 265268. doi: $10.1038 / 38460$

[13] G. Cooper, N. Kimmich, W. Belisle, et al., "Carbonaceous Meteorites as a Source of Sugar-Related Organic Compounds for the Early Earth,” Nature, Vol. 414, No. 6866, 2001, pp. 879-883. doi:10.1038/414879a

[14] P. Machalek, "Organic Molecules in Comets and Meteorites and life on Earth,” Johns Hopkins University Press. Baltymore, 2007.

[15] A. Cordova, M. Engqvist, I. Ibrahem, J. Casas, H. Sunden, "Plausible Origins of Homochirality in the Amino acid Catalyzed Neogenesis of Carbohydrates," Chemical Communications, Vol. 15, 2005, pp. 2047-2049. doi:10.1039/b500589b

[16] U. Meierhenrich, G. Muñoz, J. Hendrik, E. Jessberger and W. Thiemann, "Identification of Diamino Acids in the Murchison Meteorite," Proceedings of the National Academy of Sciences of the United States of America, Vol. 101, No. 25, 2004, pp. 9182-9186.

[17] P. Schmitt, G. Zelimir and D. G. Régis, "High Molecular Diversity of Extraterrestrial Organic Matter in Murchison Meteorite Revealed 40 Years after Its Fall,” Proceedings of the National Academy of Sciences of the United States of America, Vol. 107, No. 7, 2010, pp. 2763-2768. doi:10.1073/pnas.0912157107

[18] S. Airieau, J. Farquhar, M. Thiemens, L. Leshin, H. Bao and E. Young, "Planetesimal Sulfate and Aqueous Alteration in CM and CI Carbonaceous Chondrites," Geochemica et Cosmochimica Acta, Vol. 69, No. 16, 2005, pp. 4166-4171. doi:10.1016/j.gca.2005.01.029

[19] G. Howes, "Researcher Conducts Supercomputer Simulations to Learn Impacts on Earth’s Magnetic Field,” 2013. http://phys.org/news/2013-04-supercomputer-simulations -impacts-earth-magnetic.html 